\title{
Index autorum ad Vol. 168
}

Confecit: C. Loeb-Schürch (B) = Book Reviews - Buchbesprechungen - Livres nouveaux

Algvere, P. 97 Altenbrunn, H. J. 392 Anastasi, G. 109 Aspinall, P. A. 128 Auricchio, G. 475

Barry, D. R. 316 Bastiaensen, L. A. K. 325 Becker, S. C. 160(B) Bedford, M. A. 238 (B) Bialas, B. 52 Bisceglia, H. 370 Bitonte, J. L. 238 (B) Blodi, F. C. 239 (B) Bouzas, A. 360

Cambie, E. 81, 161 Capella, J. A. 324(B) Cernea, P. 13 Chishti, M. I. 197 Cioli, S. 21 Clark, B. A. J. 58 Constantin, Florica 13

De Laey, J. J., v. Laey, J. J. de Dernouchamps, J. P. 132 De Rouck, A., v. Rouck, A. de Dietze, U. 230 Dobree, J. H. 77 (B) Dobrescu, Gioconda 13 Dujovny, M. 261

Edelhauser, H. F. 324(B) El-Sayed, M. E. 39 El-Shewy, T. M. 39

Francois, J. 81, 122, 161, 288, 299, 417 Freyler, H. 400 Friedman, A. H. 197

Gartner, J. 1 Gasset, A. R. 238 (B) Gault, I. G. 154 Gierek, Ariadna 52 Girardier, L. 140

Gjötterberg, M. 97 Glosckner, S. L. 239(B) Goes, F. 299 Guerra, R. 193

Harris, L. S. 221

Havener, W. H. 78 (B), 239 (B)

Henkind, P. 197

Heremans, J. F. 132

Hommes, O. R. 325

Horn, D. L. van 324 (B)

Hughes, Joan 221

Irinoda, K. 76(B) Israeli, J. 261

Jabloński, J. 27 Jung, R. 324(B)

492

Index autorum

Karel,I. 446 Kaufmann, H. E. 238 (B) Keates, R. H. 238 (B) Krieglstein, G. K. 188 Kurz, O. 241 Laey, J.J.de 161,288 Lauricella, M. 475 Lerche,W. 206 Lewitus, Z. 241 Linnert, D. 188

Lommatzsch, P. 376, 392 Lowe, R. F. 58

Madroszkiewicz, M. 47, 462 Manor, R. S. 241,261,269 Michiels, J. 132 Molinelli, G. 193

Reiner, J. 78 (B)

Romeo, G. 109

Rouck, A. de 81, 161

Rutgers v. d. Loeff-Manschot, W. 178

Sakic, D. 253

Salama Benarroch, A. R. de 370

Salama Benarroch, I. 370

Santoro, A. 109

Sarnat, B. G. 154

Schroder, K.-D. 230

Scullica,L. 109 
Shaffer, R. N. 76(B)

Shapiro, D. 366

Shimmyo, M. 221

Sobańska, Maria 465

Sosnierz, Marian 52

Stadhouders, A. M. 325

Nano, H. 370 Neckel, J. 206 Neetens, A. 122

Otto, A. J. 308

Peleska, M. 446 Perez, H. 370 Perkins, E. S. 77 (B) Pilz, A. 376 Pinckers, A. 178,308 Ponte, F. 475 Puff,K.-H. 77(B) Pülhorn, G. 348 Puzzolo, D. 109

Tasman,W. 76(B) Thiel,H.-J. 348 Thijssen, J. M. 308 Tilgner, S. 230 Tosi, P. 193 Tsacopoulos, M. 140

Ulrich,W.-D. 376

Vaerman, J. P. 132 Van Horn, D. L., v Votocková, J. 446 Vuagnat, P. 140

Weiss, D.I. 76(B)

Horn, D. L. van

R. Radnót, Magda 282

Zlatar, P. 253 